\title{
ON SOME HERMITIAN FORMS ASSOCIATED WITH TWO GIVEN CURVES OF THE COMPLEX PLANE*
}

\author{
BY \\ GABRIEL SZEGŐ
}

1. Let us consider the set of polynomials $f(z)$ of degree $n$ satisfying the condition $|f(z)| \leqq 1$ on a given closed set $E$. What can then be said about $|f(z)|$ on another given closed set $E^{\prime}$ ?

The maximum of $|f(z)|$ on $E^{\prime}$ for the set of all polynomials defined by the inequality mentioned is a number $m_{n}=m_{n}\left(E, E^{\prime}\right)$ which depends on $n$ and also on the mutual position of $E$ and $E^{\prime}$. It is of course an invariant with respect to translation and rotation. Obviously $m_{n}=1$ if $E^{\prime}$ is a subset of $E$. For the sake of simplicity we further assume that $E$ and $E^{\prime}$ are closed regions bounded by rectifiable Jordan curves $C$ and $C^{\prime}$. In case $C^{\prime}$ has some points exterior to $C$, the exact determination of $m_{n}$ seems to be rather difficult. The asymptotic calculation for large values of $n$, however, is possible. Fekete [1] has shown the existence of

$$
\lim _{n \rightarrow \infty}\left(m_{n}\right)^{1 / n}=\rho .
$$

Here the number $\rho$ can be characterized by means of the conformal and oneto-one representation of the region exterior to $C$ onto the region exterior to a circle in the $w$-plane, $z=\infty$ corresponding to $w=\infty$. The points of $C^{\prime}$ outside of $C$ have some correspondents in the w-plane. The greatest distance of these from the center of the circle, divided by the radius of the circle, is equal to $\rho$.

2. An analogous problem can be proposed if the maximum value of $|f(z)|$ on $C$ is replaced by its "mean quadratic value,"

$$
\left\{l^{-1} \int_{C}|f(z)|^{2}|d z|\right\}^{1 / 2}
$$

here $C$ is supposed to have the length $l$. A similar definition of the deviation on $C^{\prime}$ may be used. The corresponding maximum $M_{n}=M_{n}\left(C, C^{\prime}\right)$ depends again on the mutual position of $C$ and $C^{\prime}$. On introducing the normalized orthogonal polynomials $\left\{q_{\nu}(z)\right\}$ associated with the curve $C[8]$ it can be easily shown that

\footnotetext{
* Presented to the Society, April 10, 1936; received by the editors February 24, 1936.
} 


$$
M_{n}^{2}=\max \sum_{\mu, \nu=0}^{n} x_{\mu} \bar{x}_{\nu}\left(l^{\prime}\right)^{-1} \int_{C^{\prime}} q_{\mu}(z) \overline{q_{\nu}(z)}|d z|,
$$

provided that the variables $x_{\nu}$ satisfy the condition $\sum_{\nu=0}^{n}\left|x_{\nu}\right|^{2} \leqq 1$ (or $=1$ ). In other words, $M_{n}^{2}$ is the greatest characteristic value of the Hermitian form on the right side of (2). Here $l^{\prime}$ denotes the length of $C^{\prime}$.

3. In the present paper we shall not enter into the details of this general problem. [The answer which can be expected is, incidentally, the same as in (1).] Only a special case shall be treated here, in which $C$ is the real interval $-1,+1$ (actually a limiting case of a Jordan curve) and $C^{\prime}$ the unit circle with the origin as center. We derive then, for the corresponding number $M_{n}$ the following asymptotic expression:*

$$
M_{n} \cong 2^{-9 / 8} \pi^{-3 / 4} n^{-1 / 4}\left(2^{1 / 2}+1\right)^{n+3 / 2} .
$$

It can be easily shown, that in this case $\rho=2^{1 / 2}+1, \rho$ having the same meaning as above. Another formulation of this result is: Let $\lambda_{n}$ be the smallest characteristic value of the quadratic form

$$
\frac{1}{2} \int_{-1}^{1}\left(x_{0}+x_{1} t+x_{2} t^{2}+\cdots+x_{n} t^{n}\right)^{2} d t=\sum_{\mu, \nu=0}^{n} \frac{x_{\mu} x_{\nu}}{\mu+\nu+1},
$$

where $\sum^{\prime}$ indicates that the summation is extended only over even values of $\mu+\nu$. We have then

$$
\lambda_{n} \cong 2^{9 / 4} \pi^{3 / 2} n^{1 / 2}\left(2^{1 / 2}-1\right)^{2 n+3},
$$

since $\lambda_{n}=M_{n}^{-2}$.

In a letter on November 19, 1935, Professor Tamarkin proposed the problem of determining an asymptotic expression for these numbers $\lambda_{n}$. I dealt with this question several years ago in connection with some investigations about the so-called Hankel forms [7]. I found at that time instead of (5), the less sharp result (18) of Part I. The letter of Professor Tamarkin gave me the opportunity to attack this problem again and to obtain the more exact formula (5) as well as the subsequent related results.

The reader may be interested in the facts previously known about the form (4). Hilbert [3] obtained an explicit expression for its determinant

$$
D_{n}=2^{n(n+1)} \frac{(1 ! 2 ! \cdots n !)^{4}}{1 ! 2 ! 3 ! \cdots(2 n+1) !} .
$$

Moreover, it is known $\dagger$ that the greatest characteristic value $\Lambda_{n}$ tends to $\pi / 2$

* In what follows, we write $a_{n} \cong b_{n}\left(a_{n}\right.$ and $b_{n}$ real or complex, $\left.\neq 0\right)$ if $\lim _{n \rightarrow \infty} a_{n} b_{n}^{-1}=1$; furthermore, $a_{n} \sim b_{n}\left(a_{n}\right.$ and $b_{n}$ positive) if two positive constants $u$ and $v$ exist such that $u<a_{n} b_{n}^{-1}<v$.

$\dagger$ See, for instance, G. H. Hardy, J. E. Littlewood, and G. Pólya, Inequalities, Cambridge, 1934, p. 226. 
if $n \rightarrow \infty$. It would be extremely interesting to find a simple way of describing asymptotically the distribution of all characteristic values of this form for large values of $n$.

4. If the range $(-1,+1)$ is replaced by another segment, similar results are valid; the asymptotic behavior of the numbers corresponding to $\lambda_{n}$ depends, of course, on the situation of this segment. For instance, in case of the form

$$
\int_{0}^{1}\left(x_{0}+x_{1} t+x_{2} t^{2}+\cdots+x_{n} t^{n}\right)^{2} d t=\sum_{\mu, \nu=0}^{n} \frac{x_{\mu} x_{\nu}}{\mu+\nu+1}
$$

we obtain for the smallest characteristic value

$$
\lambda_{n} \cong 2^{15 / 4} \pi^{3 / 2} n^{1 / 2}\left(2^{1 / 2}-1\right)^{4 n+4} .
$$

[We have in this case $\rho=\left(2^{1 / 2}+1\right)^{2}$.] The greatest characteristic value tends here to $\pi$.

5. For infinite sets, the integrals considered are not convergent. Thus the introduction of a proper weight factor becomes necessary. The special cases

$$
\int_{-\infty}^{\infty} e^{-t^{2}}\left(x_{0}+x_{1} t+x_{2} t^{2}+\cdots+x_{n} t^{n}\right)^{2} d t=\sum_{\mu, \nu=0}^{n} \Gamma\left(\frac{1}{2}(\mu+\nu+1)\right) x_{\mu} x_{\nu}
$$

and

$$
\int_{0}^{\infty} e^{-t}\left(x_{0}+x_{1} t+x_{2} t^{2}+\cdots+x_{n} t^{n}\right)^{2} d t=\sum_{\mu, \nu=0}^{n}(\mu+\nu) ! x_{\mu} x_{\nu}
$$

illustrate the totally different behavior of the smallest characteristic value with which we are here concerned. In these cases the corresponding asymptotical values are:*

$$
2^{13 / 4} \pi^{3 / 2} e n^{1 / 4} \exp \left(-2(2 n)^{1 / 2}\right) \text { and } 2^{3 / 2} \pi^{3 / 2} e n^{1 / 4} \exp \left(-4 n^{1 / 2}\right) .
$$

6. In Part I we start with the discussion of the form (4) proving that $\lambda_{n} \sim n^{1 / 2}\left(2^{1 / 2}-1\right)^{2 n}$. We give in Part II the proof of the sharper formula (5). In Part III the analogous treatment of the form (7) follows. Finally, we discuss the forms (9) and (10), giving the proof of (11). The formulas (5) and (8) are connected with asymptotic properties of Legendre polynomials; in cases (9) and (10) we need some asymptotic properties of Hermite and Laguerre polynomials.

\section{PART I}

1. Obviously we have in case of the form (4)

* It is easy to show that the corresponding greatest characteristic values are $\cong \Gamma\left(n+\frac{1}{2}\right)$ and $(2 n)$ !. 


$$
\lambda_{n}=\min \frac{\frac{1}{2} \int_{-1}^{1}(f(t))^{2} d t}{(1 /(2 \pi)) \int_{-\pi}^{\pi}\left|f\left(e^{i \phi}\right)\right|^{2} d \phi},
$$

or what is the same,

$$
\pi \lambda_{n}^{-1}=\max \frac{\int_{-\pi}^{\pi}\left|f\left(e^{i \phi}\right)\right|^{2} d \phi}{\int_{-1}^{1}(f(t))^{2} d t},
$$

$f(z)$ being an arbitrary polynomial of the $n$th degree with real coefficients. Supposing that $z$ lies in the complex plane cut along the segment $(-1,+1)$, we have

$$
\left(z+\left(z^{2}-1\right)^{1 / 2}\right)^{-2 n-1}(f(z))^{2}=\frac{1}{2 \pi i} \int\left(t+\left(t^{2}-1\right)^{1 / 2}\right)^{-2 n-1}(f(t))^{2} \frac{d t}{t-z},
$$

where the integral is taken in negative sense along a closed curve enclosing the segment $(-1,+1)$ but not the point $z$. The left-hand member is indeed regular in the cut plane and vanishes for $z=\infty$. By $z+\left(z^{2}-1\right)^{1 / 2}$ we mean that branch of the function which is $\infty$ for $z=\infty$. For this branch $\left|z+\left(z^{2}-1\right)^{1 / 2}\right|>1$ in the cut plane and $=1$ on the cut itself.

Thus we get

$$
\left|z+\left(z^{2}-1\right)^{1 / 2}\right|^{-2 n-1}|f(z)|^{2} \leqq \frac{1}{\pi \delta} \int_{-1}^{1}(f(t))^{2} d t,
$$

$\delta$ being the shortest distance of $z$ from $(-1,+1)$.

Another inequality, less exact in general, can be obtained using some elementary facts about Legendre polynomials. We have [Pólya-Szegö, 5, vol. 2, p. 95, problem 103]

$$
\left|\left(z+\left(z^{2}-1\right)^{1 / 2}\right)^{-2 n}(f(z))^{2}\right| \leqq \max _{-1 \leqq t \leqq+1}(f(t))^{2} \leqq \frac{1}{2}(n+1)^{2} \int_{-1}^{1}(f(t))^{2} d t .
$$

2. The unit circle $|z|=1$ lies entirely interior to the ellipse $\left|z+\left(z^{2}-1\right)^{1 / 2}\right|$ $=1+2^{1 / 2}$ having with it only the points $\pm i$ in common. Let $\gamma$ be an arc $\pi / 2-\epsilon \leqq \phi \leqq \pi / 2+\epsilon$ of the unit circle containing $+i$ and lying in the upper half plane; let $\gamma^{\prime}$ be the complementary arc of the upper half circle. Then we have on account of (13)

$$
\int_{\gamma}|f(z)|^{2} d \phi \leqq \int_{\gamma}\left|z+\left(z^{2}-1\right)^{1 / 2}\right|^{2 n+1} d \phi \cdot \frac{1}{\pi \cos \epsilon} \int_{-1}^{1}(f(t))^{2} d t .
$$

On setting $z=e^{i \phi}=i e^{i \phi^{\prime}}$ we have in the neighborhood of $z=i$, i.e., for small values of $\phi^{\prime}$, 


$$
\begin{aligned}
& \log \left[z+\left(z^{2}-1\right)^{1 / 2}\right]=\log \left[i e^{i \phi^{\prime}}+i\left(e^{2 i \phi^{\prime}}+1\right)^{1 / 2}\right] \\
& =\frac{i \pi}{2}+\log \left[e^{i \phi^{\prime}}+e^{i \phi^{\prime} / 2}\left(2 \cos \phi^{\prime}\right)^{1 / 2}\right] \\
& =\frac{i \pi}{2}+\log \left\{1+i \phi^{\prime}-\frac{\phi^{\prime 2}}{2}+\cdots\right. \\
& \left.\quad+2^{1 / 2}\left(1+i \frac{\phi^{\prime}}{2}-\frac{\phi^{\prime 2}}{8}+\cdots\right)\left(1-\frac{\phi^{\prime 2}}{4}+\cdots\right)\right\} \\
& =\frac{i \pi}{2}+\log \left(1+2^{1 / 2}\right)+\log \left\{1+i \frac{\phi^{\prime}}{2^{1 / 2}}-\left(2^{1 / 2}+2\right) \frac{\phi^{\prime 2}}{8}+\cdots\right\} \\
& =\frac{i \pi}{2}+\log \left(1+2^{1 / 2}\right)+2^{-1 / 2} i \phi^{\prime}-2^{-1 / 2} \frac{\phi^{\prime 2}}{4}+\cdots,
\end{aligned}
$$

whence

$$
\begin{aligned}
\left|z+\left(z^{2}-1\right)^{1 / 2}\right| & =\exp \left\{\Re \log \left(z+\left(z^{2}-1\right)^{1 / 2}\right)\right\} \\
& =\left(2^{1 / 2}+1\right) \exp \left\{-2^{-1 / 2} \frac{\phi^{\prime 2}}{4}+\cdots\right\} .
\end{aligned}
$$

Using a familiar argument [cf. Pólya-Szegö, 5, vol. 1, p. 78, problem 201] we obtain

$$
\int_{\gamma}\left|z+\left(z^{2}-1\right)^{1 / 2}\right|^{2 n+1} d \phi \sim n^{-1 / 2}\left(2^{1 / 2}+1\right)^{2 n}
$$

and on account of (15)

$$
\frac{\int_{\gamma}|f(z)|^{2} d \phi}{\int_{-1}^{1}(f(t))^{2} d t}=O\left(n^{-1 / 2}\left(2^{1 / 2}+1\right)^{2 n}\right) .
$$

3. On the other hand we obtain on $\gamma^{\prime}$ using (14)

$$
\frac{|f(z)|^{2}}{\int_{-1}^{1}(f(t))^{2} d t} \leqq \frac{1}{2}(n+1)^{2} \lambda^{2 n},
$$

$\lambda$ being the maximum of $\left|z+\left(z^{2}-1\right)^{1 / 2}\right|$ on $\gamma^{\prime}$. But $\lambda<2^{1 / 2}+1$, whence

follows.

$$
\frac{\int_{-\pi}^{\pi}\left|f\left(e^{i \phi}\right)\right|^{2} d \phi}{\int_{-1}^{1}(f(t))^{2} d t}=O\left(n^{-1 / 2}\left(2^{1 / 2}+1\right)^{2 n}\right)
$$

4. For the special polynomial $f(t)=f(\cos \theta)=\cos n \theta$

$$
\int_{-1}^{1}(f(t))^{2} d t=\int_{0}^{\pi} \cos ^{2} n \theta \sin \theta d \theta=1-\left(4 n^{2}-1\right)^{-1}
$$


holds. Furthermore

$$
\int_{-\pi}^{\pi}\left|f\left(e^{i \phi}\right)\right|^{2} d \phi=\int_{-\pi}^{\pi}\left|\frac{1}{2}\left(z+\left(z^{2}-1\right)^{1 / 2}\right)^{n}+\frac{1}{2}\left(z-\left(z^{2}-1\right)^{1 / 2}\right)^{n}\right|_{z=e^{i \phi}}^{2} d \phi .
$$

The contribution of $\gamma^{\prime}$ to this integral is $=O\left(\lambda^{n}\right)$. On the other hand on $\gamma$

$$
\begin{aligned}
\frac{1}{4} \int_{\gamma}\left|z+\left(z^{2}-1\right)^{1 / 2}\right| 2 n\left|1+\left(z-\left(z^{2}-1\right)^{1 / 2}\right)^{-2 n}\right|^{2} d \phi \\
>\frac{1}{4}\left(1-\lambda^{-2 n}\right)^{2} \int_{\gamma}\left|z+\left(z^{2}-1\right)^{1 / 2}\right|^{2 n} d \phi .
\end{aligned}
$$

According to (17) the last expression is $>c n^{-1 / 2}\left(2^{1 / 2}+1\right)^{2 n}$, where $c>0$ is independent of $n$. Thus we have shown

$$
\begin{gathered}
\lambda_{n} \sim n^{1 / 2}\left(2^{1 / 2}-1\right)^{2 n} . \\
\text { PART II }
\end{gathered}
$$

1. In order to prove the more precise statement (5), we introduce the normalized Legendre polynomials $q_{n}(z)=\left(n+\frac{1}{2}\right)^{1 / 2} P_{n}(z), P_{n}$ being the ordinary Legendre polynomial of the $n$th degree. On writing $f(z)$ as a linear combination of Legendre polynomials we obtain

$$
\pi \lambda_{n}^{-1}=\max \sum_{\mu, \nu=0}^{n} K_{\mu \nu} x_{\mu} \bar{x}_{\nu} \quad \text { for } \quad \sum_{\nu=0}^{n}\left|x_{\nu}\right|^{2} \leqq 1,
$$

where

$$
K_{\mu \nu}=\int_{-\pi}^{\pi} q_{\mu}(z) \overline{q_{\nu}(z)} d \phi, \quad z=e^{i \phi}
$$

According as $n$ is even or odd, Legendre polynomials are even or odd functions, so that $K_{\mu \nu}=0$ if $\mu-\nu$ is odd. Furthermore, the term $z^{n-2 k}$ in $P_{n}(z)$ has the sign $(-1)^{k}$; consequently $K_{\mu \nu}$ is real and has the $\operatorname{sign}(-1)^{(\mu-\nu) / 2}=e^{i \pi(\mu-\nu) / 2}$ if $\mu-\nu$ is even. Thus the maximum problem (19) can be separated into the two independent problems

$$
\begin{cases}\max \sum_{\mu, \nu=0}^{n}{ }^{(1)} K_{\mu \nu} x_{\mu} \bar{x}_{\nu}, & \sum_{\nu \equiv n}\left|x_{\nu}\right|^{2} \leqq 1, \\ \max \sum_{\mu, \nu=0}^{n}{ }^{(2)} K_{\mu \nu} x_{\mu} \bar{x}_{\nu}, & \sum_{\nu \equiv n-1}\left|x_{\nu}\right|^{2} \leqq 1,\end{cases}
$$

where, in $\sum^{(1)}, \mu \equiv \nu \equiv n(\bmod 2)$, while, in $\sum^{(2)}, \mu \equiv \nu \equiv n-1(\bmod 2)$, and $\pi \lambda_{n}^{-1}$ is the greater of the corresponding maximum values. 
2. We now propose to study some asymptotic properties of the integrals (20) using the asymptotic formula of Heine [2, p. 174]

$$
P_{n}(z) \cong \frac{\left(z+\left(z^{2}-1\right)^{1 / 2}\right)^{n+1 / 2}}{(2 \pi n)^{1 / 2}\left(z^{2}-1\right)^{1 / 4}}
$$

Here $z$ lies in the complex plane cut along the segment $(-1,+1)$. The ratio of the expressions in (22) tends to 1 uniformly in every closed region having no points in common with the segment $(-1,+1)$.

Let $\omega$ be a fixed positive integer, $n \rightarrow \infty$. We confine ourselves to the values of $\mu$ and $\nu$ characterized by the conditions

$$
n-\omega \leqq \mu, \nu \leqq n, \quad \mu-\nu \text { even. }
$$

Denoting again by $\gamma$, as in Part I, the $\operatorname{arc} \pi / 2-\epsilon \leqq \phi \leqq \pi / 2+\epsilon$ and using (16), we have for $z=e^{i \phi}=i e^{i \phi^{\prime}}$,

$$
\begin{aligned}
& \int_{\gamma} q_{\mu}(z) \overline{q_{\nu}(z)} d \phi \\
& \cong\left(\mu+\frac{1}{2}\right)^{1 / 2}\left(\nu+\frac{1}{2}\right)^{1 / 2} \int_{\gamma} \frac{\left(z+\left(z^{2}-1\right)^{1 / 2}\right)^{\mu+1 / 2}\left(z+\left(z^{2}-1\right)^{1 / 2}\right)^{\nu+1 / 2}}{(2 \mu \pi)^{1 / 2}(2 \nu \pi)^{1 / 2}\left|\left(z^{2}-1\right)^{1 / 4}\right|^{2}} d \phi \\
& \cong \exp \frac{i \pi(\mu-\nu)}{2} \frac{2^{-1 / 2}\left(2^{1 / 2}+1\right)^{\mu+\nu+1}}{2 \pi} \\
& \cdot \int_{-\epsilon}^{e} \exp \left\{\left(2^{-1 / 2} i \phi^{\prime}-2^{-1 / 2} \frac{\phi^{\prime 2}}{4}+\cdots\right)\left(\mu+\frac{1}{2}\right)\right. \\
& \cong(-1)^{(\mu-\nu) / 2} \frac{2^{-1 / 2}\left(2^{1 / 2}+1\right)^{\mu+\nu+1}}{2 \pi} \\
&\left.\left.\cdot \int_{-\epsilon}^{\epsilon} \exp \left\{2^{-1 / 2} i(\mu-\nu) \phi^{\prime}-2^{-1 / 2}(\mu+\nu+1) \frac{\phi^{\prime 2}}{4}+\cdots\right\} d \phi^{\prime}-2^{-1 / 2} \frac{\phi^{\prime 2}}{4}+\cdots\right)\left(\nu+\frac{1}{2}\right)\right\} d \phi^{\prime} \\
& \cong(-1)^{(\mu-\nu) / 2} \frac{2^{-1 / 2}\left(2^{1 / 2}+1\right)^{\mu+\nu+1}}{2 \pi} \int_{-\epsilon}^{e} \exp \left\{-2^{-1 / 2}(\mu+\nu+1) \frac{\phi^{\prime 2}}{4}\right\} d \phi^{\prime} \\
& \cong(-1)^{(\mu-\nu) / 2} \frac{2^{-1 / 2}\left(2^{1 / 2}+1\right)^{\mu+\nu+1}}{2 \pi}\left\{2^{-1 / 2} \frac{\mu+\nu+1}{4}\right\}^{-1 / 2} \pi^{1 / 2} \\
& \cong(-1)^{(\mu-\nu) / 2}\left(2 \pi 2^{1 / 2}\right)^{-1 / 2} n^{-1 / 2}\left(2^{1 / 2}+1\right)^{\mu+\nu+1},
\end{aligned}
$$

and therefore

$$
K_{\mu \nu} \cong(-1)^{(\mu-\nu) / 2} 2^{1 / 4} \pi^{-1 / 2} n^{-1 / 2}\left(2^{1 / 2}+1\right)^{\mu+\nu+1} .
$$


Using Schwarz's inequality we obtain for all values of $\mu$ and $\nu$

$$
\left|K_{\mu \nu}\right| \leqq K_{\mu \mu}^{1 / 2} K_{\nu \nu}^{1 / 2} \text {. }
$$

On the other hand, for the values of $\mu$ and $\nu$ satisfying (23) on account of (24),

$$
K_{\mu \nu} \cong(-1)^{(\mu-\nu) / 2} K_{\mu \mu}^{1 / 2} K_{\nu \nu}^{1 / 2} .
$$

3. Now if $\left\{\epsilon_{\nu}\right\}$ denotes a suitable sequence with positive terms $\rightarrow 0$,

$$
\begin{aligned}
\sum_{\mu, \nu=0}^{n} K_{\mu \nu}^{(1)} x_{\mu} \bar{x}_{\nu} & \leqq \sum\left|K_{\mu \nu}\right|\left|x_{\mu}\right|\left|x_{\nu}\right| \leqq \sum K_{\mu \mu}^{1 / 2} K_{\nu \nu}^{1 / 2}\left|x_{\mu}\right|\left|x_{\nu}\right| \\
& =\left\{\sum_{\nu \equiv n} K_{\nu \nu}^{1 / 2}\left|x_{\nu}\right|\right\}^{2} \leqq \sum_{\nu=n} K_{\nu \nu} \\
& \leqq 2^{1 / 4} \pi^{-1 / 2}\left(2^{1 / 2}+1\right)^{2 n+1} \sum_{\nu=n}\left(1+\epsilon_{\nu}\right) \nu^{-1 / 2}\left(2^{1 / 2}+1\right)^{2 \nu-2 n} \\
& \cong 2^{1 / 4} \pi^{-1 / 2}\left(2^{1 / 2}+1\right)^{2 n+1} n^{-1 / 2} \sum_{k=0}^{\infty}\left(2^{1 / 2}+1\right)^{-4 k} \\
& =2^{-9 / 4} \pi^{-1 / 2} n^{-1 / 2}\left(2^{1 / 2}+1\right)^{2 n+3}
\end{aligned}
$$

On the other hand we put

$$
\begin{array}{llrl}
x_{\nu}=\sigma e^{i \pi \nu / 2} K_{\nu \nu}^{1 / 2} & \text { if } \nu \equiv n, & n-\omega \leqq \nu \leqq n, \\
x_{\nu}=0 & \text { if } \nu \equiv n, & \nu<n-\omega .
\end{array}
$$

Here the positive number $\sigma$ has to be determined according to the condition $\sum_{\nu \equiv n}\left|x_{\nu}\right|^{2}=\sigma^{2} \sum_{n-\omega \leqq \nu \leqq n ; \nu \equiv n} K_{\nu \nu}=1$. The form in question becomes then

$$
\sum_{\substack{\mu, v=n-\omega \\ \mu=n=n}}^{n} K_{\mu \nu} \sigma^{2} \exp [i \pi(\mu-\nu) / 2] K_{\mu \mu}^{1 / 2} K_{\nu \nu}^{1 / 2}
$$

In view of $\left(25^{\prime}\right)$ we obtain for this expression

$$
\begin{aligned}
& \cong \sigma^{2} \sum K_{\mu \mu} K_{\nu \nu}=\sigma^{2}\left\{\sum K_{\nu v}\right\}^{2}=\sum_{\substack{n=n-\omega \\
\nu=n}}^{n} K_{\nu \nu} \\
& \cong 2^{1 / 4} \pi^{-1 / 2} n^{-1 / 2}\left(2^{1 / 2}+1\right)^{2 n+1} \sum_{\substack{v=n-\omega \\
\nu=n}}^{n}\left(2^{1 / 2}+1\right)^{2 \nu-2 n} .
\end{aligned}
$$

Since the number $\omega$ is arbitrarily large the asymptotic value of the first maximum (21) is given by the right-hand member in (26). The second maximum is obtained by replacing here $n$ by $n-1$; the result is of lower order than the preceding one which therefore furnishes the asymptotic evaluation of (19). 


\section{PART III}

The treatment of the form (7) does not present new difficulties. Using notations analogous to those employed in Part II, it is sufficient to point out only the essential differences.

The orthogonal polynomials belonging to the interval $(0,1)$ are

$$
q_{n}(t)=(2 n+1)^{1 / 2} P_{n}(2 t-1)=(2 n+1)^{1 / 2} \sum_{k=0}^{n} \frac{P_{n}^{(k)}(-1)}{k !}(2 t)^{k} .
$$

Hence we obtain

$$
K_{\iota \nu}=(2 \mu+1)^{1 / 2}(2 \nu+1)^{1 / 2} \int_{-\pi}^{\pi} P_{\mu}(2 z-1) \overline{P_{\nu}(2 z-1)} d \phi, \quad z=e^{i \phi} .
$$

Since $\operatorname{sgn} P_{n}{ }^{(k)}(-1)=(-1)^{n-k}$ we easily see that $K_{\mu \nu}$ is real and has the sign $(-1)^{\mu-\nu}$. The inequality (25) holds again.

In this case, $z=e^{i \phi}$,

$$
\max \left|2 z-1+\left[(2 z-1)^{2}-1\right]^{1 / 2}\right|=3+2\left(2^{1 / 2}\right)
$$

and this value is attained for $z=-1$. Thus the main part of our integrals is given by the arc $\pi-\epsilon \leqq \phi \leqq \pi+\epsilon$. We need therefore, the development of

$$
\log \left\{2 z-1+\left[(2 z-1)^{2}-1\right]^{1 / 2}\right\}=2 \cdot \log \left\{z^{1 / 2}+\left(\left(z^{1 / 2}\right)^{2}-1\right)^{1 / 2}\right\}
$$

if $z=e^{i \phi}=-e^{i \phi^{\prime}}$ and $\phi^{\prime}$ is small. Since $z^{1 / 2}=i e^{i \phi^{\prime} / 2}$ this can be readily calculated from (16) in the form

$$
i \pi+2 \log \left(2^{1 / 2}+1\right)+2^{-1 / 2} i \phi^{\prime}-2^{1 / 2} \frac{\phi^{\prime 2}}{16}+\cdots .
$$

This yields under the condition

$$
n-\omega \leqq \mu, \nu \leqq n
$$

the asymptotic formula

$$
\begin{aligned}
K_{\mu \nu} \cong & \left.(2 \mu+1)^{1 / 2}(2 \nu+1)^{1 / 2} \int_{\pi-\epsilon}^{\pi+\epsilon} P_{\mu}(2 z-1) \overline{P_{\nu}(2 z-1}\right) d \phi \\
\cong & (2 \mu+1)^{1 / 2}(2 \nu+1)^{1 / 2} \\
& \int_{\pi-\epsilon}^{\pi+\epsilon} \frac{\left\{2 z-1+\left((2 z-1)^{2}-1\right)^{1 / 2}\right\}^{\mu+1 / 2}\left\{\overline{\left.2 z-1+\left((2 z-1)^{2}-1\right)^{1 / 2}\right\}^{\nu+1 / 2}}\right.}{(2 \pi \mu)^{1 / 2}(2 \pi \nu)^{1 / 2}\left|\left((2 z-1)^{2}-1\right)^{1 / 4}\right|^{2}} d \phi \\
\cong & (-1)^{\mu-\nu}\left(2 \pi 2^{1 / 2}\right)^{-1}\left(2^{1 / 2}+1\right)^{2 \mu+2 \nu+2} \\
& \int_{-\epsilon}^{+\epsilon} \exp \left\{2^{-1 / 2} i(\mu-\nu) \phi^{\prime}-2^{1 / 2}(\mu+\nu+1) \frac{\phi^{\prime 2}}{16}\right\} d \phi^{\prime}
\end{aligned}
$$




$$
\begin{aligned}
& \simeq(-1)^{\mu-\nu}\left(2 \pi 2^{1 / 2}\right)^{-1}\left(2^{1 / 2}+1\right)^{2 \mu+2 \nu+2}\left\{\frac{2^{1 / 2}(\mu+\nu+1)}{16}\right\}^{-1 / 2} \pi^{1 / 2} \\
& \simeq(-1)^{\mu-\nu} 2^{-1 / 4} \pi^{-1 / 2} n^{-1 / 2}\left(2^{1 / 2}+1\right)^{2 \mu+2 \nu+2} .
\end{aligned}
$$

For the values $(31)$ the asymptotic formula $\left(25^{\prime}\right)$ holds again, with $(-1)^{(\mu-\nu) / 2}$ replaced by $(-1)^{\mu-\nu}$.

Consequently, by means of an argument similar to that used in Part II, we obtain for

$$
\begin{gathered}
2 \pi \lambda_{n}^{-1}=\max \sum K_{\mu \nu} x_{\mu} \bar{x}_{\nu}, \quad \sum\left|x_{\nu}\right|^{2} \leqq 1 \\
2 \pi \lambda_{n}^{-1} \cong \sum_{\nu=0}^{n} K_{\nu \nu} \cong 2^{-1 / 4} \pi^{-1 / 2} n^{-1 / 2}\left(2^{1 / 2}+1\right)^{4 n+2} \sum_{k=0}^{\infty}\left(2^{1 / 2}+1\right)^{-4 k} \\
=2^{-11 / 4} \pi^{-1 / 2} n^{-1 / 2}\left(2^{1 / 2}+1\right)^{4 n+4} .
\end{gathered}
$$

\section{PART IV}

1. Introducing the normalized Hermite polynomials $q_{n}(t)$ belonging to the weight function $e^{-t^{2}}$, we see that the smallest characteristic value $\lambda_{n}$ of (9) is given by

$$
2 \pi \lambda_{n}^{-1}=\max \sum K_{\mu \nu} x_{\mu} \bar{x}_{\nu}, \quad \sum\left|x_{\nu}\right|^{2} \leqq 1,
$$

where

$$
K_{\mu \nu}=\int_{-\pi}^{\pi} q_{\mu}(z) \overline{q_{\nu}(z)} d \phi, \quad z=e^{i \phi} .
$$

Hermite polynomials can be represented easily in terms of Laguerre polynomials with the parameter values $\pm \frac{1}{2}$. Using the notation from the book by Pólya-Szegö [5, vol. 2, p. 94, problem 99] we obtain [cf. loc. cit., p. 95, problem 102]

$$
q_{n}(t)= \begin{cases}\left(\Gamma\left(m+\frac{1}{2}\right)\right)^{-1 / 2}(\Gamma(m+1))^{1 / 2} L_{m}^{(-1 / 2)}\left(t^{2}\right) & \text { if } n=2 m \\ \left(\Gamma\left(m+\frac{s}{2}\right)\right)^{-1 / 2}(\Gamma(m+1))^{1 / 2} t L_{m}^{(1 / 2)}\left(t^{2}\right) & \text { if } n=2 m+1 .\end{cases}
$$

Consequently $K_{\mu \nu}=0$ if $\mu-\nu$ is odd. For $\mu-\nu$ even, $K_{\mu \nu}$ is real and positive. According to the asymptotic formula of Laguerre polynomials due to Perron [cf. 4, p. 77, (48)]

$$
q_{n}(z) \cong \eta\left(2 \pi^{1 / 2}\right)^{-1}\left(\frac{n}{2}\right)^{-1 / 4} \exp \left[z^{2} / 2-i z(2 n)^{1 / 2}\right]
$$

where $\eta=1$ if $n$ is even, $\eta=i$ if $n$ is odd. Here $z$ is supposed to have a positive imaginary part. The essential part of $K_{\mu \nu}$ is given again by an arc $\gamma$ of the 
type of Part II.* Let $\omega$ be a fixed positive number; for large values of $n$ and for

$$
n-\omega n^{1 / 2} \leqq \mu, \nu \leqq n, \quad \mu-\nu \text { even, }
$$

we have

$$
\begin{aligned}
& \int_{\gamma} q_{\mu}(z) \overline{q_{\nu}(z)} d \phi \\
& \quad \cong(4 \pi e)^{-1}\left(\frac{\mu}{2}\right)^{-1 / 4}\left(\frac{\nu}{2}\right)^{-1 / 4} \int_{\gamma} \exp \left[-i z(2 \mu)^{1 / 2}+i \bar{z}(2 \nu)^{1 / 2}\right] d \phi .
\end{aligned}
$$

Putting $z=i e^{i \phi^{\prime}}$ we obtain

$$
\begin{aligned}
& (4 \pi e)^{-1}\left(\frac{n}{2}\right)^{-1 / 2} \int_{-e}^{e} \exp \left\{\left(1+i \phi^{\prime}-\frac{\phi^{\prime 2}}{2}\right)(2 \mu)^{1 / 2}\right. \\
& \left.\quad+\left(1-i \phi^{\prime}-\frac{\phi^{\prime 2}}{2}\right)(2 \nu)^{1 / 2}\right\} d \phi^{\prime} \\
& \cong(4 \pi e)^{-1}\left(\frac{n}{2}\right)^{-1 / 2} \exp \left[(2 \mu)^{1 / 2}+(2 \nu)^{1 / 2}\right] \\
& \quad \cdot \int_{-\epsilon}^{\epsilon} \exp \left[-\frac{1}{2}\left((2 \mu)^{1 / 2}+(2 \nu)^{1 / 2}\right) \phi^{\prime 2}\right] d \phi^{\prime} \\
& \cong(4 \pi e)^{-1}\left(\frac{n}{2}\right)^{-1 / 2} \exp \left[(2 \mu)^{1 / 2}+(2 \nu)^{1 / 2}\right]\left\{\frac{1}{2}\left((2 \mu)^{1 / 2}+(2 \nu)^{1 / 2}\right)\right\}^{-1 / 2} \pi^{1 / 2} \\
& \cong 2^{-7 / 4} \pi^{-1 / 2} e^{-1} n^{-3 / 4} \exp \left[(2 \mu)^{1 / 2}+(2 \nu)^{1 / 2}\right]
\end{aligned}
$$

because $\mu^{1 / 2}-\nu^{1 / 2}$ remains bounded. Hence, for the values of $\mu, \nu$ defined by (36)

$$
K_{\mu \nu} \cong 2^{-3 / 4} \pi^{-1 / 2} e^{-1} n^{-3 / 4} \exp \left[(2 \mu)^{1 / 2}+(2 \nu)^{1 / 2}\right] .
$$

Hence we get as in Part II

$$
\begin{aligned}
& 2 \pi \lambda_{n}^{-1} \cong \sum_{\nu \equiv n} K_{\nu \nu} \cong 2^{-3 / 4} \pi^{-1 / 2} e^{-1} n^{-3 / 4} \sum_{\nu \equiv n} \exp \left[2(2 \nu)^{1 / 2}\right] \\
& \cong 2^{-3 / 4} \pi^{-1 / 2} e^{-1} n^{-3 / 4} \cdot \frac{1}{2} \int_{1}^{n} \exp \left[2(2 x)^{1 / 2}\right] d x \\
&=2^{-3 / 4} \pi^{-1 / 2} e^{-1} n^{-3 / 4} \int_{1}^{n^{1 / 2}} x \exp \left[2\left(2^{1 / 2}\right) x\right] d x \cong 2^{-9 / 4} \pi^{-1 / 2} e^{-1} n^{-1 / 4} \exp \left[2(2 n)^{1 / 2}\right]
\end{aligned}
$$

Thus the first statement (11) is established.

2. Dealing with the form (10) we use Laguerre polynomials $q_{n}(t)=L_{n}(t)$.

* Near the real axis, i.e., in the neighborhood of the points $z= \pm 1$, the formula (35) cannot be used. Here the fact has to be regarded that $\left|q_{n}(x+i y)\right|, x$ and $y$ real, is monotonically increasing if $|y|$ increases. This is a consequence of the reality of the zeros of $q_{n}(z)$. 
Here $K_{\mu \nu}$ is always real and positive. Excluding the case in which $z$ is real and non-negative, we have [Perron, 4 , loc. cit.]

$$
q_{n}(z) \cong\left(2 \pi^{1 / 2}\right)^{-1}(-z)^{-1 / 4} e^{z / 2} n^{-1 / 4} \exp \left[2(-z n)^{1 / 2}\right]
$$

The essential part of $K_{\mu \nu}$ is given now by the contribution of a neighborhood of $z=-1$ (cf. the footnote on p. 460).

Restricting $\mu$ and $\nu$ as in (36) but without the condition regarding $\mu-\nu$ we obtain

$$
\begin{aligned}
K_{\mu \nu} & \cong \int_{\pi-\epsilon}^{\pi+\epsilon} q_{\mu}(z) \overline{q_{\nu}(z)} d \phi \\
& \cong(4 \pi e)^{-1} \mu^{-1 / 4} \nu^{-1 / 4} \int_{\pi-\epsilon}^{\pi+\epsilon} \exp \left[2(-z \mu)^{1 / 2}+2(-\bar{z} \nu)^{1 / 2}\right] d \phi .
\end{aligned}
$$

Writing $z=-e^{i \phi^{\prime}}$ we get

$$
\begin{aligned}
& (4 \pi e)^{-1} n^{-1 / 2} \int_{-\epsilon}^{+\epsilon} \exp \left\{2 \mu^{1 / 2}\left(1+i \frac{\phi^{\prime}}{2}-\frac{\phi^{\prime 2}}{8}\right)+2 \nu^{1 / 2}\left(1-i \frac{\phi^{\prime}}{2}-\frac{\phi^{\prime 2}}{8}\right)\right\} d \phi^{\prime} \\
& \cong(4 \pi e)^{-1} n^{-1 / 2} \exp \left[2 \mu^{1 / 2}+2 \nu^{1 / 2}\right] \int_{-e}^{\int} \exp \left[-\left(\mu^{1 / 2}+\nu^{1 / 2}\right) \phi^{\prime 2} / 4\right] d \phi^{\prime} \\
& \cong(4 \pi e)^{-1} n^{-1 / 2} \exp \left[2 \mu^{1 / 2}+2 \nu^{1 / 2}\right]\left\{\frac{\mu^{1 / 2}+\nu^{1 / 2}}{4}\right\}^{-1 / 2} \pi^{1 / 2} \\
& \cong 2^{-3 / 2} \pi^{-1 / 2} e^{-1} n^{-3 / 4} \exp \left[2 \mu^{1 / 2}+2 \nu^{1 / 2}\right]
\end{aligned}
$$

therefore

$$
2 \pi \lambda_{n}^{-1} \cong \sum_{n=0}^{n} K_{v v} \cong 2^{-3 / 2} \pi^{-1 / 2} e^{-1} n^{-3 / 4} \int_{1}^{n} \exp \left[4 x^{1 / 2}\right] d x \cong 2^{-1 / 2} \pi^{-1 / 2} e^{-1} n^{-1 / 4} \exp \left[4 n^{1 / 2}\right] .
$$

\section{BIBLIOGRAPHY}

1. M. Fekete, Über den absoluten Betrag von Polynomen, welche auf einer Punktmenge gleichmässig beschränkt sind, Mathematische Zeitschrift, vol. 26 (1927), pp. 324-344.

2. H. E. Heine, Handbuch der Kugelfunktionen, 2d edition, Berlin, 1878.

3. D. Hilbert, Ein Beitrag zur Theorie des Legendre'schen Polynoms, Acta Mathematica, vol. 18 (1894), pp. 155-159.

4. O. Perron, Über das Verhalten einer ausgearteten hypergeometrischen Reihe bei unbegrenztem Wachstum eines Parameters, Journal für Mathematik, vol. 151 (1921), pp. 63-78.

5. G. Polya-G. Szegö, A ufgaben und Lehrsätze aus der Analysis, 2 volumes, Berlin, 1925.

6. I. Schur, Bemerkungen zur Theorie der beschränkten Bilinearformen mit unendlich vielen Veränderlichen, Journal für Mathematik, vol. 140 (1911), pp. 1-28.

7. G. Szegö, A Hankel-féle formákról, Matematikai és Természettudományi Értesítö, vol. 36 (1918), pp. 497-538.

8. G. Szegö, Über orthogonale Polynome, die zu einer gegebenen Kurve der komplexen Ebene gehören, Mathematische Zeitschrift, vol. 9 (1921), pp. 218-270.

WASHINGTON UNIVERSITY,

St. Louis, Mo. 